Tecnología y

Ciencias $₫$ Agua
2019, Instituto Mexicano de Tecnología del

Agua.Open Access bajo la licencia CC BY-NC-SA 4.0

(https://creativecommons.org/licenses/by-nc-

$\mathrm{sa} / 4.0 /)$

DOI: $10.24850 /$ j-tyca-2019-05-12

Artículos

\title{
Estado del arte: una revisión actual a los mecanismos que realizan los humedales artificiales para la remoción de nitrógeno y fósforo
}

\section{State of art: A current review of the mechanisms that make the artificial wetlands for the removal of nitrogen and phosphorus}

Viviana M. Arteaga-Cortez ${ }^{1}$

Abel Quevedo-Nolasco ${ }^{2}$

David $\mathrm{H}$. del Valle-Paniagua ${ }^{3}$

Martiniano Castro-Popoca ${ }^{4}$

Ángel Bravo-Vinaja ${ }^{5}$

Jorge A. Ramírez-Zierold ${ }^{6}$

${ }^{1}$ Colegio de Postgraduados, Campus Montecillo, carretera MéxicoTexcoco km 36.5, Estado de México, México, varteaga@colpos.mx, ORCID: 0000-0002-3002-6063

${ }^{2}$ Colegio de Postgraduados, Campus Montecillo, carretera MéxicoTexcoco km 36.5, Estado de México, México, anolasco@colpos.mx

${ }^{3}$ Colegio de Postgraduados, Campus Montecillo, carretera MéxicoTexcoco km 36.5, Estado de México, México, dhvallep@colpos.mx, ORCID: 0000-0003-4383-4323

${ }^{4}$ Colegio de Postgraduados, Campus Montecillo, carretera MéxicoTexcoco km 36.5, Estado de México, México, mcastro@colpos.mx ${ }^{5}$ Maestría en Innovación en el Manejo de Recursos Naturales, Campus San Luis Potosí, Colegio de Postgraduados, San Luis Potosí, México, abravo@colpos.mx, ORCID: 0000-0001-8806-0275 
Tecnología y

Ciencias $₫$ Agua
2019, Instituto Mexicano de Tecnología del

Agua.Open Access bajo la licencia CC BY-NC-SA 4.0

(https://creativecommons.org/licenses/by-nc-

$\mathrm{sa} / 4.0 /)$

${ }^{6}$ Posgrado en Ciencias del Mar y Limnología, Universidad Nacional Autónoma de México, Ciudad Universitaria, Ciudad de México, México, jramirezzierold@yahoo.com.mx, ORCID: 0000-0001-8094-1852

Autor para correspondencia: Viviana M. Arteaga-Cortez, varteaga@colpos.mx

\title{
Resumen
}

El tratamiento de aguas residuales por medio de los humedales artificiales se utiliza como una tecnología verde que surge de la necesidad de replicar los beneficios ecosistémicos de los humedales naturales para tratar diversos afluentes de aguas residuales. Este trabajo presenta una revisión bibliográfica acerca de los diferentes tipos de humedales artificiales y sus procesos depurativos. Si bien existen muchos estudios sobre humedales artificiales, es necesario realizar una sinergia de dicha información. No obstante, aunque los humedales artificiales son una frecuente práctica de gestión para la mitigación de contaminantes provenientes de escorrentía agrícola y municipal, en muchos países faltan estudios comparativos sobre su eficiencia depurativa y el mantenimiento a los mismos, así como profundizar en la investigación de sustratos alternativos y detallar medidas preventivas para la evitar la colmatación del sustrato.

Palabras clave: tratamiento de aguas residuales, humedales construidos, remoción, nitrógeno, fósforo.

\begin{abstract}
The wastewater treatment through artificial wetlands is used as a green technology, arising from a need to replicate the eco-systemic benefits of natural wetlands, to treat various effluents of wastewater. This work presents a bibliographic review about the different types of artificial wetlands and their depurative processes. Even though there are many studies on artificial wetlands, the information, so that is necessarily make to a synergy of this information. Although artificial wetlands are a frequent management practice for the mitigation of pollutants from agricultural and municipal runoff, in many countries, comparative studies on their purification efficiencies, their mantainance, as well as in
\end{abstract}


Tecnología y

Ciencias $₫$ Agua
2019, Instituto Mexicano de Tecnología del

Agua.Open Access bajo la licencia CC BY-NC-SA 4.0 (https://creativecommons.org/licenses/by-nc-

$\mathrm{sa} / 4.0 /)$

deepen the investigation of alternative substrates, and preventive measures to avoid the clogging of the substrate, are needed.

Keywords: Wastewater treatment, constructed wetlands, removal, nitrogen, phosphorus.

Recibido: 22/02/2017

Aceptado: 26/04/2019

\section{Introducción}

Un grave problema en la actualidad es la contaminación de corrientes y cuerpos de agua, los cuales son receptores de vertidos de carácter doméstico, industrial y agrícola, vertiéndose directamente sin ningún tipo de tratamiento a las corrientes naturales y los cuerpos de agua (Cloter, Mazari, \& De Anda, 2006).

El problema es el detrimento de la calidad del agua de los lagos y embalses que presentan niveles importantes de concentración de materia orgánica, sólidos suspendidos, sólidos disueltos, nutrimentos, compuestos tóxicos y microorganismos de interés sanitario. Los vertidos de las industrias agroalimentarias, zonas rurales, urbanas y turísticas se caracterizan por el aporte de altas cargas orgánicas. Por esto, el reúso de estas aguas para riego agrícola trae como consecuencia riesgos colaterales para la salud de la población, en función de las concentraciones de contaminantes (Ramírez-Carrillo, Luna-Pabello, \& Arredondo-Figueroa, 2009). Estas aguas residuales exigen tratamientos, que en su mayoría son costosos y con alto impacto ambiental en su construcción e implementación. En tal sentido, la gestión de aguas residuales tanto en zonas rurales como urbanas constituye un reto debido tanto a la falta de infraestructura adecuada como capacidad económica. 
Ciencias
2019, Instituto Mexicano de Tecnología del

Agua.Open Access bajo la licencia CC BY-NC-SA 4.0

(https://creativecommons.org/licenses/by-nc-

$\mathrm{sa} / 4.0 /)$

El tratamiento de las aguas residuales es vital y va encaminado al uso de ecotecnologías o tecnologías adecuadas que utilicen o imiten a la naturaleza, y así aprovechar algunos componentes de la misma, como biota y suelos. Los humedales artificiales son sistemas específicamente diseñados y construidos por el hombre para el tratamiento de aguas residuales, donde se aumentan las capacidades depuradoras de la vegetación y con ello la eficacia del sistema, al optimizar los procesos físicos, químicos y biológicos que ocurren en los ecosistemas de humedales naturales (Vymazal \& Brix, 1998). La remoción de nitrógeno inicia con la transformación del nitrógeno orgánico $\left(\mathrm{N}_{\mathrm{Org}}\right)$ a nitrógeno

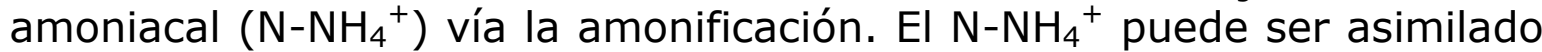
por las raíces de las plantas o los microorganismos, volatilizarse si el sistema presenta un $\mathrm{pH}$ superior a 8 o bien oxidarse a nitrato $\left(\mathrm{NO}_{3}{ }^{-}\right)$ (nitrificación) por acción de las bacterias amonio-oxidantes y nitritooxidantes. El último paso es la reducción del $\mathrm{NO}_{3}{ }^{-}$a nitrógeno molecular $\left(\mathrm{N}_{2}\right)$ bajo condiciones anóxicas/anaerobias (desnitrificación) (TorresBojorges, Zurita, \& Tejeda, 2017).

Con la finalidad de depurar altas cargas orgánicas surgen los humedales denominados humedales artificiales de alta tasa (HAAT), los cuales utilizan como sustrato elementos de plástico, consiguiendo alta porosidad para tratar aguas residuales. La diversidad de vegetación es un factor importante respecto a la capacidad de eliminación de contaminantes en humedales. La rizósfera de las macrófitas estimula el crecimiento de las comunidades microbianas, por lo tanto, cualquier modificación de la diversidad de la planta y su hábitat lleva a cambios en la comunidad microbiana, y consecuentemente a la eficiencia de remoción de contaminantes (Corzo, Sanabria, \& García, 2016).

El objetivo principal de este documento es revisar y discutir acerca del estado actual de los humedales artificiales, los distintos tipos de humedales, y sobre todo la tecnología dirigida a la eliminación de nitrógeno y materia orgánica.

\section{Materiales y métodos}


Teçnología y

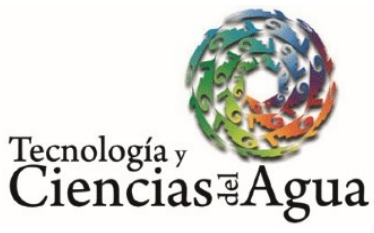

2019, Instituto Mexicano de Tecnología del

Agua.Open Access bajo la licencia CC BY-NC-SA 4.0

(https://creativecommons.org/licenses/by-nc-

sa/4.0/)

Se realizó una búsqueda exhaustiva de literatura para recopilar estudios que proporcionaran mayor información sobre "constructed wetlands". Elsevier, Ebsco, Springer, Science Citation Index Expanded (SCIE) y Social Sciences Citation Index (SSCI) del Web of Science (WOS) y Scopus fueron las bases de datos utilizadas para la recopilación de tales estudios. Los resultados de la búsqueda en línea comprenden estudios realizados hasta el $1^{\circ}$ de agosto de 2018.

\section{Humedales naturales y artificiales}

Se denomina humedal natural a todas aquellas áreas que están inundadas por aguas dulces o saladas, de forma temporal o permanente, y que presentan una vegetación típica adaptada para vivir en condiciones de suelo saturado (Vymazal \& Brix, 1998). Los ecosistemas ribereños actúan como sumidero de nutrimentos para escurrimiento de tierras altas $y$, por lo tanto, estos humedales son zonas de amortiguamiento importantes entre tierras agrícolas y arroyos. Existen algunas técnicas de mitigación más comúnmente utilizadas en el campo para prevenir la entrada de plaguicidas en los cuerpos de agua, como los bordes de campo; barreras vegetativas (franjas de vegetación densa, que funcionan como una barrera porosa filtrante para el agua de escorrentía) (Vymazal \& Březinová, 2015); zanjas con vegetación, y humedales artificiales (HA). Los humedales artificiales proporcionan una superficie para el crecimiento de los microorganismos encargados de la filtración y adsorción de los contaminantes, además de inhibir el crecimiento de algas.

Los humedales artificiales se clasifican en humedales de flujo libre o sistemas de plantas de libre flotación (FL), y humedales subsuperficiales o sistemas de plantas con raíces emergentes (FSS), los cuales se dividen a su vez en sistemas de flujo vertical (FSSV) y sistemas de flujo horizontal (FSSH). 
Tecnologí y
Ciencias
2019, Instituto Mexicano de Tecnología del

Agua.Open Access bajo la licencia CC BY-NC-SA 4.0

(https://creativecommons.org/licenses/by-nc-

$\mathrm{sa} / 4.0 /)$

Los humedales de flujo libre o superficial son estanques o canales en los que la superficie del agua se encuentra expuesta a la atmósfera y contienen plantas enraizadas flotantes. Por otro lado, los humedales de flujo subsuperficial son estanques en los cuales se coloca un sustrato poroso, el cual puede ser arena, grava o cualquier tipo de suelo de textura gruesa, el cual presenta vegetación emergente. Son de dos clases: humedales de flujo horizontal y flujo vertical, donde cambia la forma de suministro del afluente al humedal. La alimentación de los humedales de flujo vertical se realiza por intervalos de tiempo, para permitir la percolación del afluente en el sustrato.

Si bien el uso de los humedales artificiales es para diversos tipos de aguas residuales, se documentan como métodos naturales en el tratamiento de aguas desde 1912. La Agencia de Protección Ambiental de los Estados Unidos (EPA, 1993) fue la primera en realizar un muestreo en el periodo comprendido de los años 1960 a 1970. De igual forma, también se documentan los proyectos realizados en todo el mundo de 1972 a 1983; a partir de 1970 se inician los estudios sobre la depuración de nitrógeno y fósforo ( $\mathrm{N}$ y $\mathrm{P}$ ) por medio de los humedales artificiales. Asimismo, en la década de 1970 se estudian los humedales construidos con la finalidad de depurar pesticidas. Durante el decenio de 1990 se estudió la depuración de nutrimentos por medio de humedales artificiales ubicados en ríos (Vymazal \& Březinová, 2015).

En 1967 se desarrolla un sistema de flujo libre a escala. En Alemania, en la década de 1970, el Dr. Kichuth creó un sistema llamado "Método de zona de raíces", el cual consistía en un humedal de flujo horizontal, en el que sembró $P$. australis, añadiendo la combinación de calcio, y hierro o aluminio para provocar la precipitación del fósforo. En el caso del estudio de los humedales en EUA, el desarrollo fue con base en los resultados de los trabajos europeos, mediante el estudio de los humedales naturales. Esta ecotecnología también fue estudiada por la NASA, que desarrolló su propio sistema, al cual Ilamaron "Sistema de tratamiento híbrido" debido a que utilizaba microrganismos anaerobios y plantas emergentes ( $P$. communis) (Rodríguez, 2003).

\section{Ventajas y desventajas}


Tecnología y

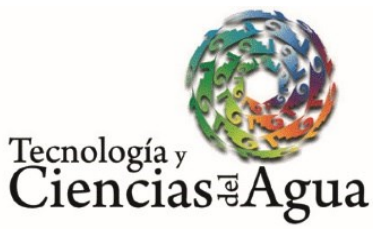

2019, Instituto Mexicano de Tecnología del

Agua.Open Access bajo la licencia CC BY-NC-SA 4.0

(https://creativecommons.org/licenses/by-nc-

$\mathrm{sa} / 4.0 /)$

Los humedales artificiales presentan grandes ventajas técnicas, económicas, sociales y estéticas sobre tratamientos convencionales de aguas residuales, ya que permiten lograr una buena calidad del agua tratada conforme a la normatividad vigente y apta para ser usada en el riego agrícola debido a que elimina microorganismos patógenos. Los costos de implementación, operación y mantenimiento, en comparación con los sistemas tradicionales de tratamiento de aguas residuales son menores, pues tienen una vida útil de 25 años y sus requerimientos energéticos de operación son mínimos, dado que la conducción del agua a los humedales es prácticamente por gravedad. Debido a las características de construcción de los humedales, es difícil que tengan desequilibrios por el cambio en las concentraciones de los contaminantes o la oscilación en el afluente del humedal (Miranda \& Luna-Pabello, 2001). Además, en estos sistemas, donde el agua fluye por debajo de la superficie del medio, no se presentan problemas con el desarrollo de mosquitos y no despiden olores, e incluso proporcionan protección térmica al sistema (vegetación, sustrato y agua), lo que hace posible la implementación de humedales artificiales en lugares donde ocurren grandes heladas (Llagas, Wilmer, \& Gómez, 2006).

A pesar de todas las ventajas que proporcionan este tipo de sistemas de depuración de agua, tienen como desventaja requerir, en general, de grandes extensiones de terreno para su implementación. Cabe señalar que los humedales con flujo subsuperficial tienen también como desventaja el costo del medio de soporte que se utiliza, así como su traslado y colocación (Rodríguez, 2003).

\section{Tipos de humedales artificiales}

La aplicación de humedales artificiales es un tema delicado debido a la variabilidad en la composición de las aguas residuales, variando con frecuencia las necesidades de tratamiento. La selección de la vegetación 
Teçnología y

Ciencias $\approx$ Agua
2019, Instituto Mexicano de Tecnología del

Agua.Open Access bajo la licencia CC BY-NC-SA 4.0

(https://creativecommons.org/licenses/by-nc-

$\mathrm{sa} / 4.0 /)$

es un tema importante en este tipo de diseños, ya que deben sobrevivir a los posibles efectos tóxicos de las aguas residuales (Calheiros, Rangel, \& Castro, 2007).

\section{Flujo libre (FL)}

Los humedales de flujo libre son quizás los más antiguos, se utilizan como tratamiento secundario y para la depuración de pesticidas. Se diseñan para cargas superficiales bajas, y con una profundidad que oscila de entre 5 a $90 \mathrm{~cm}$; sin embargo, es más común encontrarlos entre 30 y $40 \mathrm{~cm}$, conformados por zanjas de $3 \mathrm{~m}$ de ancho y $100 \mathrm{~m}$ de largo, con una profundidad que oscila entre 0.30 y $0.40 \mathrm{~m}$, donde la vegetación usada es Scirpus lacustris. La efectividad de remoción en los humedales de flujo libre es del 96\% para SST; 96\% para DBO; $87 \%$ para DQO (demanda química de oxigeno), y $30 \%$ para PT (fósforo total) (Rodríguez, 2003).

\section{Flujo subsuperficial horizontal}

Los humedales FSSH surgen como una alternativa de tratamientos secundarios. Son sistemas con medios porosos saturados, donde se siembra con vegetación emergente. Su uso abarca aguas residuales provenientes de la industria y son muy comunes para el tratamiento de las aguas residuales de procesamiento de alimentos (Vymazal, 2009).

Un humedal FSSH presenta una alta remoción de materia orgánica, $\mathrm{DBO}_{5}$ (demanda bioquímica de oxígeno medida a cinco días), DQO y sólidos suspendidos totales. Sin embargo, presentan una menor eliminación de nutrientes. Por tanto, las plantas $T$. latifolia y $P$. australis son especies vegetales que se adaptan mejor a las aguas residuales de curtidurías en términos de supervivencia y propagación. Los FSSH son 
Tecnologí y
Ciencias
2019, Instituto Mexicano de Tecnología del

Agua.Open Access bajo la licencia CC BY-NC-SA 4.0

(https://creativecommons.org/licenses/by-nc-

$\mathrm{sa} / 4.0 /)$

una alternativa viable para reducir el contenido de materia orgánica de las aguas residuales, pues toleran las fluctuaciones de flujo o intermitencias (Calheiros et al., 2007). Asimismo, funcionan como protección contra la salinización secundaria del suelo. La vegetación que se utiliza para la desalinización de sustratos agrícolas es Typha spp., Phragmites communis, Phragmites japonica, Medicago sativa Linn., Lemna minor L., Potamogeton crispus, Typha spp., Phragmites communis y Potamogeton crispus. La eficiencia de remoción de $\mathrm{Ca}^{2+}$, $\mathrm{Mg}^{2+}, \mathrm{Na}^{+} \mathrm{Cl}^{-}$y $\mathrm{SO}_{4}{ }^{2-}$ puede llegar al $80 \%$ con Typha spp. (Yang, Sheng, Zhang, Kang, \& Liu, 2015).

Los suelos arcillosos, por tener capacidad de intercambio iónico, permiten la remoción temporal del nitrógeno presente en las aguas residuales, que depende del tiempo de estabilización del sistema. En el caso de los humedales de flujo horizontal, que emplean suelo, presentan un potencial de remoción adicional de fósforo y amonio; mientras que en los sistemas de flujo vertical debido a la intermitencia del flujo favorece la restauración de las condiciones aerobias y el amonio es adsorbido. Al usar grava en humedales, la capacidad de remoción de fósforo es limitada.

Las macrófitas más usadas para el tratamiento de aguas residuales en sistemas tipo estanque es de dos clases principalmente: plantas acuáticas flotantes y sumergidas. Las plantas flotantes absorben el dióxido de carbono según sus necesidades de oxígeno, mientras que la vegetación sumergida se inhibe por la turbiedad (Yang et al., 2015).

\section{Flujo subsuperficial vertical}

La aplicación del agua en humedales subsuperficiales de flujo vertical (FSSV) se realiza de manera uniforme sobre el lecho. Por lo común se emplean como sistemas secundarios y se componen de dos etapas: una con dos celdas de flujo vertical seguido por una celda de flujo horizontal; estas últimas etapas tienen la finalidad de lograr la depuración de los efluentes. La principal ventaja de este tipo de sistemas radica en restaurar las condiciones aerobias durante periodos 
Tecnologí y
Ciencias
2019, Instituto Mexicano de Tecnología del

Agua.Open Access bajo la licencia CC BY-NC-SA 4.0

(https://creativecommons.org/licenses/by-nc-

$\mathrm{sa} / 4.0 /)$

secos (Rodríguez, 2003). La eficiencia del proceso de depuración depende en gran medida de la aireación del sustrato; además, este tipo de sistemas son susceptibles a la obstrucción, por lo cual es recomendable limitar la alimentación de carga orgánica a $25 \mathrm{~g} \mathrm{DQO} / \mathrm{m}^{2}$ por día, además de utilizar el sistema en forma rotatoria, de este modo se logra la oxigenación del lecho, y se permite que el sistema tenga un tiempo de descanso, en el cual se le permite al lecho secarse (RamírezCarrillo et al., 2009).

Los sistemas de humedales de flujo vertical con recirculación y de operación intermitente, incluso operando bajo altas cargas realizan una mejor mezcla en el lecho del humedal, acelerando la difusión de oxígeno y el aumento depurativo del $\mathrm{DQO}$ y $\mathrm{NO}_{3}{ }^{-}$, reduciendo así la superficie que se utiliza en este tipo de sistemas, favoreciendo sobre todo a los humedales construidos en regiones montañosas, donde la disponibilidad de espacio es limitada (Foladori, Ruaben, \& Ortigara, 2013).

\section{Sistemas de humedales combinados o híbridos}

Los humedales de flujo horizontal se caracterizan por mantener condiciones anóxicas, por lo que proporcionan condiciones adecuadas para la desnitrificación. En cambio, los humedales de flujo vertical mantienen condiciones aerobias, favorables para la nitrificación; de esta forma, la combinación de ambos tipos de humedales, construidos en serie, humedales híbridos, potencializan la remoción de nitrógeno total (Ntotal) (Vymazal, 2014). La combinación de estos humedales brinda una mayor eficiencia en la depuración de aguas residuales (Vymazal, 2009) en diferentes etapas del tratamiento (Torres-Bojorges et al., 2017).

Otro tipo de combinación es la realizada por los sistemas de flujo superficial con los de flujo subsuperficial, así que dependiendo de los contaminantes será la combinación óptima de estos sistemas híbridos. Vymazal (2009) hace una recopilación de la información acerca de las distintas combinaciones de sistemas híbridos, al igual que los diferentes tipos de afluentes usados para cada sistema (Tabla 1). 
Tecnología y

Ciencias $₫$ Agua
2019, Instituto Mexicano de Tecnología del

Agua.Open Access bajo la licencia CC BY-NC-SA 4.0 (https://creativecommons.org/licenses/by-nc-

$\mathrm{sa} / 4.0 /)$

Tabla 1. Sistemas híbridos de humedales artificiales. Sinopsis correspondiente al prototipo de humedal y tipo de afluente (Vymazal, 2009).

\begin{tabular}{|c|c|c|}
\hline Tipo de humedal & 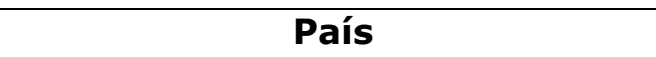 & Tipo de aguas residuales \\
\hline VF-HF & $\begin{array}{l}\text { Reino Unido, EUA, Estonia, Francia, } \\
\text { Irlanda, Túnez }\end{array}$ & Aguas residuales \\
\hline HF-VF & Dinamarca, Polonia, México & \\
\hline FL-HF & Grecia & \\
\hline HF-FL & Canadá, Kenia & \\
\hline HF-VF-HF & Polonia & \\
\hline VF-HF-FL-P & Estonia & \\
\hline VF-HF-FL-P & Tailandia & \\
\hline
\end{tabular}


Tecnología y

Ciencias $₫$ Agua
2019, Instituto Mexicano de Tecnología del

Agua.Open Access bajo la licencia CC BY-NC-SA 4.0 (https://creativecommons.org/licenses/by-nc-

$\mathrm{sa} / 4.0 /)$

\begin{tabular}{|c|c|c|}
\hline HF-VF-HF-FL & Italia & \\
\hline VF-HF & Eslovenia & \multirow{4}{*}{$\begin{array}{lll}\text { Lixiviados } & \text { de } & \text { rellenos } \\
\text { sanitarios } & & \end{array}$} \\
\hline HF-FL & Noruega, Canadá & \\
\hline FL-HF & EE.UU. & \\
\hline VF-HF-P & Portugal & \\
\hline HF-VF & Nepal & Hospital \\
\hline \multirow[t]{3}{*}{ VF-HF } & Japón & Industria lechera \\
\hline & Francia & Industria quesera \\
\hline & Tailandia & Granja de cerdo \\
\hline HF-FL & Italia & \multirow[t]{2}{*}{ Lagar } \\
\hline VF-HF-FL-P & Italia & \\
\hline \multirow[t]{5}{*}{ FL-HF } & Taiwán & Acuicultura \\
\hline & Taiwán & Acuicultura del camarón \\
\hline & Taiwán & Río contaminado \\
\hline & China & Industrial \\
\hline & Uganda & Minería \\
\hline \multirow[t]{2}{*}{ VF-HF } & Francia & Lixiviados de composta \\
\hline & Polonia & Rastro \\
\hline
\end{tabular}

$\mathrm{VF}=$ flujo vertical, $\mathrm{HF}=$ flujo horizontal, $\mathrm{FL}=$ superficie de agua libre $\circ \mathrm{P}=$ estanque.

\section{Procesos depurativos}

Los humedales artificiales se utilizan principalmente para la retención de nutrientes y materia orgánica en aguas residuales domésticas y municipales, aguas pluviales y escorrentías agrícolas. Los fertilizantes utilizados para la agricultura contienen en su mayoría fosfato, el cual infiltra las aguas subterráneas provenientes de la escorrentía de las 
Teçnología y

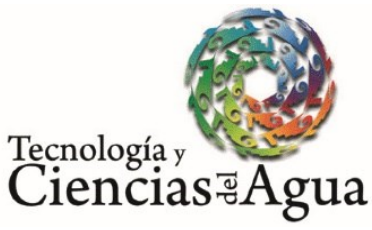

2019, Instituto Mexicano de Tecnología del

Agua.Open Access bajo la licencia CC BY-NC-SA 4.0

(https://creativecommons.org/licenses/by-nc-

$\mathrm{sa} / 4.0 /)$

tierras agrícolas (Zhang, Moon, Myneni, \& Jaffé, 2017). Si bien el P es importante para los ecosistemas, grandes cantidades pueden conducir a la eutrofización de cuerpos de agua, misma que es causada por exceso de nitrógeno $(\mathrm{N})$ y fósforo $(\mathrm{P})$, la cual origina el incremento de las algas y disminución del oxígeno disuelto en los mismos. Dentro de los humedales artificiales se efectúan procesos físicos, químicos, y biológicos, que remueven los contaminantes presentes en las aguas residuales. Entre dichos procesos se encuentran la sedimentación, adsorción a las partículas del suelo, asimilación por las plantas y transformación microbiana (Rodríguez, 2003).

Con la finalidad de favorecer el desarrollo de la biopelícula en el interior del humedal, la selección del sustrato es fundamental (Wu, Kuschk, Brix, Vymazal, \& Dong, 2014). La micro flora que se desarrolla dentro de un humedal es variada, abarca bacterias, protozoos y hasta pequeños animales, siendo las bacterias el grupo depurador fundamental en los procesos de tratamiento de aguas residuales.

El agua se mueve lentamente por el humedal debido al flujo laminar propio de estos sistemas, y la resistencia proporcionada por las raíces y la vegetación, mismas que sirven como trampas de sedimento, además de ser la ruta principal para la eliminación de fósforo (Pavlineri, Skoulikidis, \& Tsihrintzis, 2017). Si bien los humedales artificiales son eficientes para la depuración de compuestos orgánicos y sólidos en suspensión, para una mayor eliminación de nitrógeno es determinante la alimentación, tipo de humedal (Vymazal, 2018) y recirculación (Wu et al., 2014).

Los procesos biológicos de remoción en humedales es quizás el proceso más importante para la remoción de contaminantes; inician a partir de la captación de oxígeno por la planta. Algunos de los contaminantes son nutrimentos para las plantas, como los iones de nitrato, amonio y fosfato, los cuales son absorbidos por la vegetación. La remoción de los contaminantes por la vegetación puede variar en función de la edad de la planta. Las bacterias y otros microorganismos del suelo también proveen, captan y almacenan nutrimentos a corto plazo, y algunos otros contaminantes. Los microorganismos presentes en los humedales remueven compuestos orgánicos.

La depuración en este tipo de sistemas de debe por lo regular a la presencia de poblaciones microbianas adheridas a la superficie de las plantas y al medio de soporte. El metabolismo microbiano produce 
Teçnología y

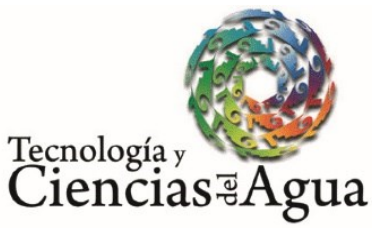

2019, Instituto Mexicano de Tecnología del

Agua.Open Access bajo la licencia CC BY-NC-SA 4.0

(https://creativecommons.org/licenses/by-nc-

$\mathrm{sa} / 4.0 /)$

también la remoción de nitrógeno inorgánico, es decir, nitrato y amonio en humedales. La remoción de nitrógeno inicia con la transformación del nitrógeno orgánico ( $\mathrm{N}_{\mathrm{Org}}$ ) a nitrógeno amoniacal $\left(\mathrm{N}-\mathrm{NH}_{4}{ }^{+}\right)$por amonificación. El $\mathrm{N}_{-} \mathrm{NH}_{4}{ }^{+}$puede ser asimilado por las raíces de las plantas o los microorganismos, volatilizarse si el sistema presenta un $\mathrm{pH}$ superior a 9 o bien oxidarse a nitrato ( $\mathrm{NO}^{-}$) por acción de las bacterias amonio-oxidantes y nitrito-oxidantes (nitrificación). El último paso es la reducción del $\mathrm{NO}^{-}$a nitrógeno molecular (N2) bajo condiciones anóxicas/anaerobias (desnitrificación). Las transformaciones que sufra el nitrógeno en los humedales dependen de su estructura, es decir, si son de flujo horizontal o vertical (Torres-Bojorges et al., 2017).

El proceso químico de remoción más importante en el suelo es la absorción, dando lugar a la retención a corto plazo y a la inmovilización a largo plazo de varias clases de contaminantes. Otro proceso importante dentro de los humedales es la adsorción, la cual se refiere a la unión de iones a las partículas del suelo a través del intercambio catiónico a la superficie de las partículas de la arcilla y de la materia orgánica del suelo.

Es a partir de la conjunción de los procesos de remoción y de los procesos metabólicos que se realizan en el humedal por la micro flora, que ocurre la remoción de los sólidos suspendidos totales (SST) y parte de la materia orgánica biodegradable (DBO) es removida a partir de la sedimentación y filtración del agua. Mientras que la materia orgánica biodegradable (DBO) se depura gracias a la degradación microbiana (aerobia, anaerobia, y facultativa). Para la eliminación del nitrógeno, los mecanismos de remoción son la amonificación, seguida de la nitrificación-desnitrificación bacteriana, la volatilización del amoniaco, además de lo que la planta consume.

El fósforo, a su vez, presenta reacciones de adsorción-precipitación con el aluminio, hierro, calcio y minerales propios de la arcilla, además de una parte que consume la planta. La remoción de los metales pesados es a través de la sedimentación y la adsorción sobre la superficie de la planta y los detritos.

Los humedales artificiales propician la remoción de patógenos mediante la sedimentación y filtración, la radiación ultravioleta, la excreción de antibióticos por las raíces de las plantas y la muerte natural (Rodríguez, 2003). La vegetación dentro de los humedales proporciona la superficie para el crecimiento microbiano, permite la filtración y la adsorción de los 
Teçnología y

Ciencias $₫$ Agua
2019, Instituto Mexicano de Tecnología del

Agua.Open Access bajo la licencia CC BY-NC-SA 4.0

(https://creativecommons.org/licenses/by-nc-

$\mathrm{sa} / 4.0 /)$

contaminantes presentes en el agua residual, inhibiendo el crecimiento de algas, y favorece la circulación del oxígeno, el cual es capturado por la vegetación a través de los tallos y las hojas, y llevado a la zona radicular para su aprovechamiento.

De igual forma, en los humedales artificiales ocurren varios procesos biogeoquímicos aeróbicos y anaeróbicos que regulan la retención y eliminación de contaminantes. El carbono y la eficiencia de eliminación de compuestos orgánicos tóxicos se da mediante la medición de oxígeno en el suelo o en el agua del efluente. La eliminación del nitrato puede comprobarse mediante la medición del carbono orgánico disuelto; en este sentido, la retención del fósforo también puede comprobarse a través de la disponibilidad del hierro reactivo y el aluminio en suelos ácidos, o bien Ca y Mg en suelos alcalinos (Reddy \& D'Angelo, 1997).

La eliminación del nitrógeno durante el tratamiento por humedales artificiales presenta procesos como volatilización de $\mathrm{NH}_{3}$, nitrificación, desnitrificación, fijación de $\mathrm{N}_{2}$, absorción de plantas y microbios, mineralización, reducción de nitrato a amonio, oxidación anaeróbica de amoniaco, fragmentación, adsorción, desorción, enterramiento, lixiviación, etcétera (Gao, Zhang, Gao, Jia, \& Yang, 2018).

La vegetación influye indirectamente en la eliminación de $\mathrm{N}$ a través de la nitrificación y desnitrificación. Dichos procesos afectan la concentración de oxígeno, en particular en la rizósfera y aumentan el suministro, al delimitar potencialmente el carbono orgánico y el nitrato a las bacterias desnitrificantes.

El transporte de oxígeno por medio de la vegetación a la rizósfera favorece los puntos de oxigenación adyacentes a la zona radicular y origina el proceso de nitrificación (Ye \& Li, 2009).

La presencia de vegetación dentro de los humedales promueve la eliminación de nutrientes, al disminuir la velocidad del flujo y aumentar la carga hidráulica, y reducir la resuspensión de los sedimentos (Laterra, Booman, Picone, Videla, \& Orúe, 2018). De la misma forma, la aireación intermitente favorece la nitrificación y desnitrificación (Llyas \& Masih, 2017).

La remoción de fósforo en los humedales inicia con el proceso de adsorción, precipitación, absorción de plantas y la acumulación de turba, fragmentación, lixiviación, mineralización y sedimentación. Sin embargo, la capacidad limitada de adsorción del sustrato y el efecto inhibidor de 
Tecnología y

Ciencias $₫$ Agua
2019, Instituto Mexicano de Tecnología del

Agua.Open Access bajo la licencia CC BY-NC-SA 4.0

(https://creativecommons.org/licenses/by-nc-

$\mathrm{sa} / 4.0 /)$

los microbios y las plantas a baja temperatura presenta serios obstáculos que impiden que los humedales artificiales eliminen $\mathrm{N}$ y $\mathrm{P}$ de manera eficiente.

La remoción de fósforo por medio de humedales artificiales se realiza de forma biológica y fisicoquímica. Si bien la interacción planta-nutriente del humedal es importante para eficientizar el proceso de purificación del cuerpo de agua eutrófico, la absorción de nutrientes por plantas y microbios dentro de los humedales es un proceso complejo, que incluye una serie de reacciones químicas y biológicas (Zhao, Li, \& Chen, 2018). Los métodos biológicos se dan a través de la asimilación del fósforo en los tejidos de la vegetación y finaliza por medio de la cosecha; mientras que en los métodos fisicoquímicos se lleva a cabo el proceso de precipitación y adsorción, el potencial de oxidación-reducción y el medio de soporte son los más importantes (Andrés, Araya, Vera, Pozo, \& Vidal, 2018).

En la actualidad hay mayor atención hacia la eliminación de plaguicidas. Tales productos son removidos en estos sistemas por procesos físicos (sedimentación, floculación, absorción, coprecipitación, precipitación); químicos (oxidación, reducción, intercambio de cationes, hidrólisis, fotólisis); biológicos (absorción y el metabolismo de la planta), o bioquímicos (degradación microbiana) (Vymazal \& Březinová, 2015). Los humedales construidos se pueden clasificar de acuerdo con diferentes criterios: hidrología (flujo superficial y flujo subsuperficial); tipos de macrófitos (flotante, emergente e sumergido), y trayectoria de flujo (horizontal o vertical) (Vymazal, 2011). Se pueden aplicar con éxito para el tratamiento descentralizado de aguas residuales en regiones con baja densidad de población (Machado, Beretta, Fragoso, \& Duarte, 2017).

En los humedales de flujo subsuperficial, el flujo de agua discurre de forma subterránea, permitiendo la oxigenación del sistema y la depuración de cargas elevadas de contaminantes orgánicos, mientras que los $\mathrm{FL}$, al igual que las IF son sistemas inundados con espejo de agua expuesto al ambiente, cuya única forma de oxigenación del sistema es la vegetación.

Las IF están condicionados al asentamiento de la vegetación por la profundidad del agua, permitiendo la acumulación de restos orgánicos de origen vegetal, animal y antrópico. 
Tecnología y

Ciencias $₫$ Agua
2019, Instituto Mexicano de Tecnología del

Agua.Open Access bajo la licencia CC BY-NC-SA 4.0 (https://creativecommons.org/licenses/by-nc-

$\mathrm{sa} / 4.0 /)$

Si bien los diferentes tipos de humedales artificiales brindan diferentes beneficios en la depuración de aguas residuales, en la Tabla 2 se realiza una comparación de las ventajas y desventajas, tanto operativas como funcionales, en la eficiencia depurativa del agua, para el nitrógeno (amoniacal, nitratos, nitritos, orgánico) y fósforo (fosfatos, orgánico) entre diferentes humedales artificiales (flujo superficial, flujo subsuperficial e islas flotantes). En humedales de flujo horizontal, la descomposición de materia orgánica es a través de procesos microbianos aeróbicos y anaeróbicos, así como por sedimentación y filtración de materia orgánica particulada; mientras que la eliminación del nitrógeno es por nitrificación, y las condiciones anóxicas y anaeróbicas son adecuadas para la desnitrificación; la eliminación del fósforo se da por adsorción y precipitación (Vymazal, 2014).

Tabla 2. Ventajas y desventajas de los mecanismos de remoción en humedales artificiales (Pavlineri et al., 2017; Vymazal, 2014; Vymazal, 2005; Foladori et al., 2013; Cooper \& Knight, 1996; Cooper, 1999).

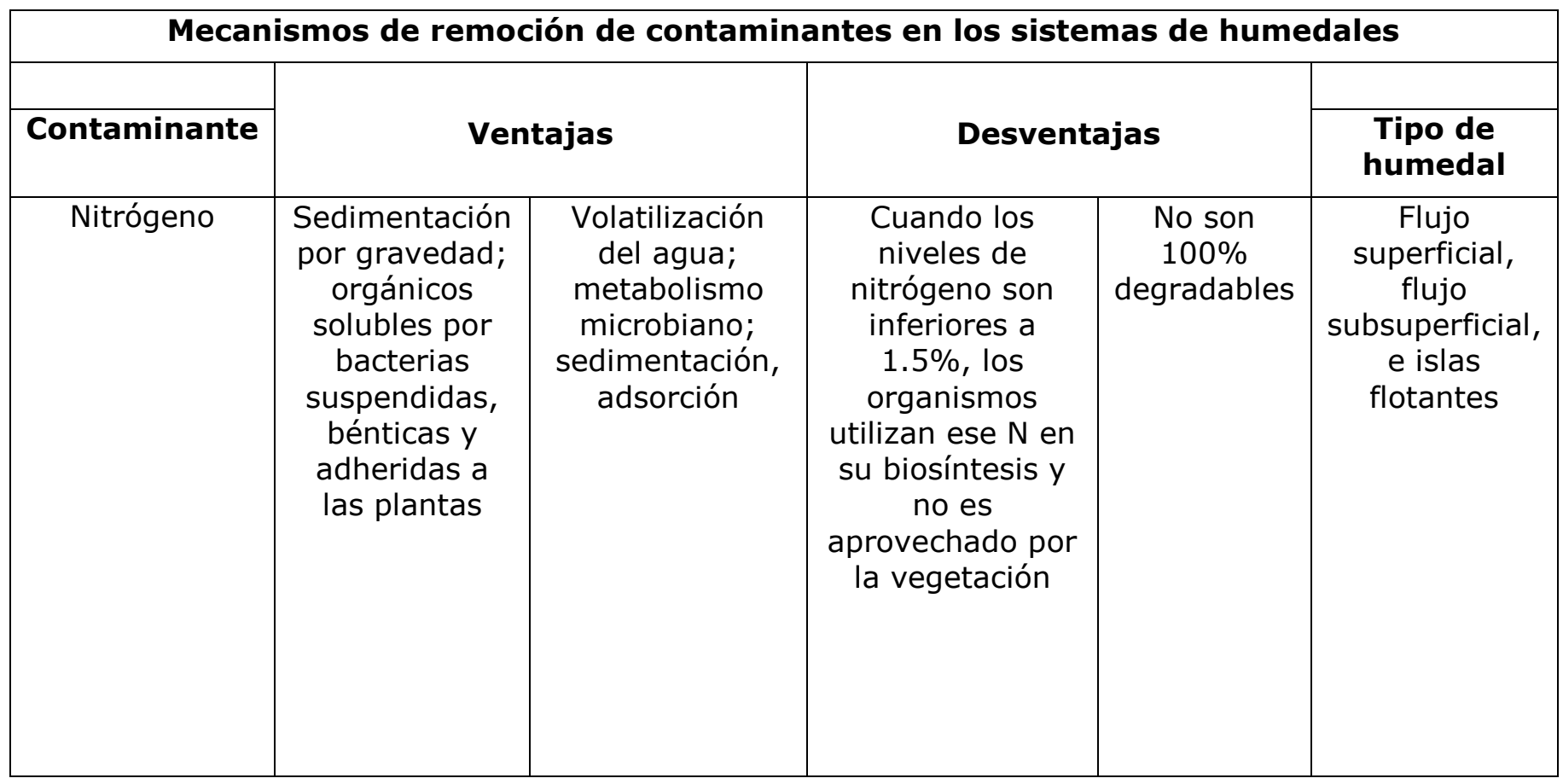


Tecnología y

Ciencias $₫$ Agua
2019, Instituto Mexicano de Tecnología del

Agua.Open Access bajo la licencia CC BY-NC-SA 4.0 (https://creativecommons.org/licenses/by-nc-

$\mathrm{sa} / 4.0 /)$

\begin{tabular}{|c|c|c|c|c|c|}
\hline $\begin{array}{l}\text { Nitrógeno } \\
\text { amoniacal }\end{array}$ & $\begin{array}{c}\text { Se presenta } \\
\text { menor } \\
\text { concentración } \\
\text { en HAFSS }\end{array}$ & $\begin{array}{c}\text { Volatilización } \\
\text { del agua, } \\
\text { nitrificación }\end{array}$ & $\begin{array}{c}\text { Se presenta } \\
\text { menor } \\
\text { concentración en } \\
\text { FL e IF }\end{array}$ & $\begin{array}{c}\text { No son } \\
100 \% \\
\text { degradables }\end{array}$ & $\begin{array}{c}\text { Remueve } \\
\text { hasta un } 94 \% \\
\text { en FV y hasta } \\
30 \% \text { en FL Y } \\
\text { HF }\end{array}$ \\
\hline Nitratos & $\begin{array}{c}\text { Amonificación, } \\
\text { nitrificación, } \\
\text { desnitrificación } \\
\text { y adsorción }\end{array}$ & $\begin{array}{l}\text { Descomposición } \\
\text { y mineralización }\end{array}$ & $\begin{array}{c}\text { El proceso } \\
\text { depende de la } \\
\text { concentración de } \\
\text { oxígeno }\end{array}$ & $\begin{array}{c}\text { No son } \\
100 \% \\
\text { degradables }\end{array}$ & $\begin{array}{c}\text { Remueve } \\
\text { hasta un 95\% } \\
\text { en FL y hasta } \\
80 \% \text { en } \\
\text { sistemas } \\
\text { combinados } \\
\text { FV + HF }\end{array}$ \\
\hline Nitritos & $\begin{array}{c}\text { Amonificación, } \\
\text { nitrificación, } \\
\text { desnitrificación } \\
\text { y adsorción }\end{array}$ & $\begin{array}{l}\text { Descomposición } \\
\text { y mineralización }\end{array}$ & $\begin{array}{c}\text { El proceso } \\
\text { depende de la } \\
\text { concentración de } \\
\text { oxígeno para su } \\
\text { remoción }\end{array}$ & $\begin{array}{c}\text { No son } \\
100 \% \\
\text { degradables }\end{array}$ & \\
\hline $\begin{array}{l}\text { Nitrógeno } \\
\text { orgánico }\end{array}$ & & $\begin{array}{c}\text { Sedimentación, } \\
\text { filtración, } \\
\text { metabolismo } \\
\text { microbiano, } \\
\text { descomposición }\end{array}$ & $\begin{array}{c}\text { El proceso } \\
\text { depende de la } \\
\text { concentración de } \\
\text { oxígeno para su } \\
\text { remoción }\end{array}$ & $\begin{array}{c}\text { No son } \\
100 \% \\
\text { degradables }\end{array}$ & \\
\hline Fósforo & $\begin{array}{c}\text { Formación o } \\
\text { coprecipitación } \\
\text { con } \\
\text { compuestos } \\
\text { insolubles }\end{array}$ & $\begin{array}{l}\text { Precipitación, } \\
\text { adsorción, } \\
\text { sedimentación }\end{array}$ & & & $\begin{array}{c}\text { Remueve } \\
\text { hasta un } 96 \% \\
\text { en FV y hasta } \\
91 \% \text { en } \\
\text { sistemas } \\
\text { combinados } \\
\text { HF + FV }\end{array}$ \\
\hline Fosfatos & & $\begin{array}{l}\text { Adsorción y } \\
\text { precipitación }\end{array}$ & $\begin{array}{c}\text { Son ligeramente } \\
\text { solubles en el } \\
\text { sustrato }\end{array}$ & & \\
\hline
\end{tabular}


Tecnología y

Ciencias $₫$ Agua
2019, Instituto Mexicano de Tecnología del

Agua.Open Access bajo la licencia CC BY-NC-SA 4.0

(https://creativecommons.org/licenses/by-nc-

$\mathrm{sa} / 4.0 /)$

\begin{tabular}{|c|c|c|c|c|}
\hline $\begin{array}{l}\text { Fósforo } \\
\text { orgánico }\end{array}$ & $\begin{array}{c}\text { Adsorción } \\
\text { sobre la } \\
\text { superficie del } \\
\text { sustrato y la } \\
\text { planta }\end{array}$ & $\begin{array}{l}\text { Sedimentación, } \\
\text { filtración, } \\
\text { metabolismo } \\
\text { microbiano, } \\
\text { descomposición, } \\
\text { adsorción }\end{array}$ & $\begin{array}{c}\text { Debe usarse } \\
\text { ósmosis inversa } \\
\text { o carbón } \\
\text { activado para su } \\
\text { remoción }\end{array}$ & $\begin{array}{c}\text { No son } \\
100 \% \\
\text { degradables }\end{array}$ \\
\hline
\end{tabular}

En humedales de flujo vertical, la difusión de oxígeno del aire contribuye mucho más a la oxigenación del lecho de filtración. A mayor oxigenación del sistema ( $>1.50 \mathrm{mg} \mathrm{OD} \mathrm{L}^{-1}$ para nitrificación y $<0.50 \mathrm{mg} \mathrm{OD} \mathrm{L}^{-1}$ para desnitrificación), mayor biodegradación y eficiencia para la remoción de nitrógeno y materia orgánica en el sistema.

La vegetación de las islas flotantes posee buena capacidad de absorción de $\mathrm{N}$ y $\mathrm{P}$; algunos de los procesos más importantes considerados en modelos acuáticos son la absorción de nutrientes por macrófitas emergentes, fitoplancton y plantas sumergidas, la alimentación del zooplancton, la mineralización de detritus, sedimentos, fósforo y sedimentos y la propagación de nutrientes, además de la difusión de fósforo y nitrógeno (Pavlineri et al., 2017; Kedlec y Wallace, 2009; Vymazal, 2013; Vymazal, 2005; Foladori et al., 2013; Cooper \& Knight, 1996).

En humedales de flujo vertical, la difusión de oxígeno del aire contribuye mucho más a la oxigenación del lecho de filtración. Con la finalidad de potencializar los beneficios que brindan los humedales tanto de flujo vertical como de flujo horizontal surgen los humedales híbridos (Vymazal, 2014).

La baja eficiencia en depuración, así como la obstrucción son problemas causados por sustratos convencionales, por lo cual las investigaciones también van encaminadas a la búsqueda de sustratos rentables y eficientes para aumentar la capacidad depuradora y minimizar la obstrucción, la concha de ostra, viruta de neumáticos, desechos de construcción y agregados de arcilla expansiva entre otros, son algunos de los materiales utilizados como sustratos. De forma ideal, los sustratos tienen propiedades fisicoquímicas únicas para la eliminación eficiente de contaminantes (Gao et al., 2018).

A mayor tiempo de retención mayor será el porcentaje de remoción de contaminantes (Ramírez-Carrillo et al., 2009). 
Tecnología y
Ciencias
2019, Instituto Mexicano de Tecnología del

Agua.Open Access bajo la licencia CC BY-NC-SA 4.0

(https://creativecommons.org/licenses/by-nc-

$\mathrm{sa} / 4.0 /)$

Con la finalidad de remover altas tasas de materia orgánica surgen como una nueva variante de humedales los denominados humedales artificiales de alta tasa (HAAT). Estos sistemas utilizan como medio de soporte el plástico, el cual favorece la formación de biofilm, consiguiendo una porosidad de hasta 94 a 96\% (Corzo et al., 2016).

Con frecuencia se usan como sustrato grava y escoria volcánica para la rehabilitación de ríos contaminados. Con el uso de escoria como sustrato se logra una mayor remoción de fósforo total (TP) y la reducción de la demanda química de oxigeno (DQO). Sin embargo, la adsorción del TP (fósforo total) por escoria volcánica se satura rápidamente durante el periodo de seguimiento ( $\mathrm{Ge}$ et al., 2015). En el sedimento de los humedales se regula la concentración de $\mathrm{P}$, adsorbiéndolo y liberándolo bajo ciertas condiciones fisicoquímicas y biológicas (Cui, Xiao Xie, \& Zhang, 2018). Para optimizar la eliminación de $\mathrm{P}$ en los humedales, se han utilizado diferentes materiales, así se permite dicho proceso a largo plazo. Zeolita, dolomita, grava, arena, caliza y apatita, alunita, lodo rojo, ceniza y escoria volcánica son algunos de los medios de soporte que se emplean dentro de los humedales para mejorar la remoción de $\mathrm{P}$ (Gao et al., 2018).

\section{Conclusiones}

Si bien existen muchos estudios sobre humedales artificiales, en la búsqueda de datos, el sesgo profesional de cada investigador se encuentra presente, por lo que es necesario realizar una sinergia de dicha información.

Los humedales artificiales son la mejor práctica de gestión para la mitigación de contaminantes (plaguicidas, fuentes no puntuales, escorrentía agrícola y drenaje) en muchos países, sin embargo aún faltan estudios comparativos sobre la eficiencia depurativa tanto de contaminantes como de humedales artificiales, así como profundizar en las ventajas y desventajas sobre el empleo de sustratos alternativos y la 
Teçnología y

Ciencias $₫$ Agua
2019, Instituto Mexicano de Tecnología del

Agua.Open Access bajo la licencia CC BY-NC-SA 4.0

(https://creativecommons.org/licenses/by-nc-

$\mathrm{sa} / 4.0 /)$

combinación de los mismos, y medidas preventivas para evitar la colmatación del sustrato dentro del humedal.

\section{Referencias}

EPA, Agencia de Protección Ambiental de Estados Unidos. (1993). Guía de diseño y construcción de un humedal construido con flujo superficial. Región 6. Dallas, EUA: Agencia de Protección Ambiental de Estados Unidos.

Andrés, E., Araya, F., Vera, I., Pozo, G., \& Vidal, G. (2018). Phosphate removal using zeolite in treatment wetlands under different oxidation-reduction potentials. Ecological Engineering, 117, 18-27.

Calheiros, C., Rangel, A., \& Castro, P. (2007). Constructed wetland systems vegetated with different plants applied to the treatment of tannery wastewater. Water Research, 41(8), 1790-1798.

Cloter, A., Mazari, H., \& De Anda, S. (2006). Atlas de la cuenca Lerma Chapala construyendo una visión conjunta. México, DF, México: Secretaría de Medio Ambiente y Recursos Naturales.

Cooper, C., \& Knight, S. (1996). Insecticide and metal contamination of a mixed cover agricultural watershed. Water Science and Technology, 33(2), 227-234.

Cooper, P. (1999). A review of the design and performance of verticalflow and hybrid reed bed treatment systems. Water Science and Technology, 40(3), 1-9.

Corzo, A., Sanabria, O., \& García, M. (2016). Optimización hidráulica de humedales artificiales de alta tasa de rellenos con medios de soporte plástico. Memorias de la III Conferencia Panamericana de Sistemas de Humedales para el Tratamiento y Mejoramiento de la calidad del Agua, 2016, Universidad Nacional del Litoral, Facultad de Ingeniería Química. Recuperado de http://www.fiq.unl.edu.ar

Cui, Y., Xiao R., Xie, Y., \& Zhang, M. (2018). Phosphorus fraction and phosphate sorption-release characteristics of the wetland sediments in the Yellow River Delta. Physics and Chemistry of the Earth, Parts A/B/C, 103, 19-27.

Foladori, P., Ruaben, J., \& Ortigara, A. (2013). Recirculation or artificial aeration in vertical flow constructed wetlands: A comparative 
Tecnología y

Ciencias $₫$ Agua
2019, Instituto Mexicano de Tecnología del

Agua.Open Access bajo la licencia CC BY-NC-SA 4.0

(https://creativecommons.org/licenses/by-nc-

$\mathrm{sa} / 4.0 /)$

study for treating high load wastewater. Bioresource Technology, 49, 398-405.

Gao, Y., Zhang, W., Gao, B., Jia, W., \& Yang, L. (2018). Highly efficient removal of nitrogen and phosphorus in an electrolysis-integrated horizontal subsurface-flow constructed wetland amended with biochar. Water Research, 139, 301-310.

Ge, Y., Wang, X., Zheng, Y., Dzakpasu, M., Zhao, Y., \& Xiong, J. (2015). Functions of slags and gravels as substrates in large-scale demonstration constructed wetland systems for polluted river water treatment Environmental Science and Pollution Research. Articles not published yet, but available online. https://doi.org/10.1007/s11356-015-4573-9

Kadlec, R. H., \& Wallace, S. (2009). Treatment wetlands ( $2^{\text {nd }}$ ed.). Boca Raton, USA: CRC Press.

Laterra, P., Booman, G., Picone, L., Videla, C., \& Orúe, M. (2018). Indicators of nutrient removal efficiency for riverine wetlands in agricultural landscapes of Argentine Pampas. Journal of Environmental Management, 222, 148-154.

Llagas, Ch., Wilmer A., \& Gómez, A. (2006). Diseño de humedales artificiales para el tratamiento de aguas residuales en la UNMSM. Revista del instituto de investigaciones FIGMMG, 15(17), 85-96.

Llyas, H., \& Masih, LI. (2017). The performance of the intensified constructed wetlands for organic matter and nitrogen removal: A review. Journal of Environmental Management, 198(1), 372-383.

Machado, A., Beretta, M., Fragoso, R., \& Duarte, E. (2017). Overview of the state of the art of constructed wetlands for decentralized wastewater management in Brazil. Journal of Environmental Management, 187, 560-570.

Miranda, M., \& Luna-Pabello, V. (2001). Estado del arte y perspectivas de aplicación de los humedales artificiales de flujo horizontal en México. Serie Tratamiento Biológica de Aguas Residuales. México, DF, México: Facultad de Química, Universidad Nacional Autónoma de México.

Pavlineri, N., Skoulikidis, N., \& Tsihrintzis, V. (2017). Constructed floating wetlands: A review of research, design, operation and 
Tecnología y

Ciencias $₫$ Agua
2019, Instituto Mexicano de Tecnología del

Agua.Open Access bajo la licencia CC BY-NC-SA 4.0 (https://creativecommons.org/licenses/by-nc$\mathrm{sa} / 4.0 /)$

management aspects, and data meta-analysis. Chemical Engineering Journal, 308, 1120-1132.

Ramírez-Carrillo, H., Luna-Pabello, V., \& Arredondo-Figueroa, J. (2009). Evaluación de un humedal artificial de flujo vertical intermitente, para obtener agua de buena calidad para la agricultura. Revista Mexicana de Ingeniería Química, 8(1), 93-99.

Reddy, K., \& D'Angelo, E. (1997). Biogeoquimical indicators to evaluate pollutant removal efficiency in constructed wetlands. Ciencia $y$ Tecnología del Agua, 35(5), 1-10.

Rodríguez, C. (2003). Humedales construidos. Estado del arte (II). México. Ingeniería hidráulica y ambiental, 24(3), 42-48.

Torres-Bojorges, A., Zurita, F., \& Tejeda, A. (2017). Carbamazepine removal in three pilot-scale hybrid wetlands planted with ornamental species. Ecological Engineering, 98, 410-417.

Vymazal, J., \& Brix, H., (1998). Constructed wetlands for waste treatment in Europe (pp. 1-15). Leiden, Netherlands: Ed. Backhuys Publishers.

Vymazal, J. (2005). Horizontal sub-surface flow and hybrid constructed wetlands systems for wastewater treatment. Ecological Engineering, 25(5), 478-490.

Vymazal, J. (2009). The use constructed wetlands with horizontal subsurface flow for various types of wastewater. Ecological Engineering, 35(1), 1-17.

Vymazal, J. (2009). The use constructed wetlands with horizontal subsurface flow for various types of wastewater. Ecological Engineering, 35(1), 1-17, https://doi.org/10.1016/j.ecoleng.2008.08.016

Vymazal, J. (2011). Constructed wetlands for wastewater treatment: Five decades of experience. Environmental Science \& Technology, 45, 61-69.

Vymazal, J. (2013). The use of hybrid constructed wetlands for wastewater treatment with special attention to nitrogen removal: A review of a recent development. Water Research, 14, 47954811. 
Tecnología y

Ciencias $₫$ Agua
2019, Instituto Mexicano de Tecnología del

Agua.Open Access bajo la licencia CC BY-NC-SA 4.0 (https://creativecommons.org/licenses/by-nc-

$\mathrm{sa} / 4.0 /)$

Vymazal, J. (2014). Constructed wetlands for treatment of industrial wastewaters: A review. Ecological Engineering, 73, 724-751.

Vymazal, J., \& Březinová, T. (2015). The use constructed wetlands with horizontal sub-surface flow for various types of wastewater. Environment International, 75(2), 11-20.

Vymazal, J. (2018). Constructed wetlands for wastewater treatment. Reference module in Earth Systems and environmental sciences. In: Encyclopedia of Ecology (pp. 765-776). Recuperado de https://books.google.com.mx/books?hl=es\&lr=\&id=x1h7DwAAQB $A J \& o i=$ fnd \&pg $=P A 14 \& d q=$ vymazal,$+2018 .+$ Constructed + wetlands + for+wastewater+treatment. +\&ots $=\mathrm{cq}-$

7vKw3Ed\&sig=bc1kisW6XNUD75x_4XZEC_rdO0M\#v=onepage \&q= vymazal\%2C\%202018.\%20Constructed\%20wetlands $\% 20$ for $\% 20$ wastewater\%20treatment. \&f $=$ false

Wu, S., Kuschk, P., Brix, H., Vymazal, V., \& Dong, R. (2014). Development of constructed wetlands in performance intensifications for wastewater treatment: A nitrogen and organic matter targeted review. Water Research, 57, 40-55.

Yang, Y., Sheng, Q., Zhang, L., Kang, H. Q., \& Liu, Y. (2015). Desalination of saline farmland drainage water through wetland plants. Agricultural Water Management, 156(7), 19-29.

Ye, F., \& Li, Y. (2009). Enhancement of nitrogen removal in towery hybrid constructed wetland to treat domestic wastewater for small rural communities. Ecological Engineering, 35, 1043-1050.

Zhang, Z., Moon, H., Myneni, S., \& Jaffé, P. (2017). Phosphate enhanced abiotic and biotic arsenic mobilization in the wetland rhizosphere. Research article. Chemosphere, 187, 130-139.

Zhao, Z., Li, Q., \& Chen, L. (2018). Effect of rhizosphere dispersal and impulsive input on the growth of wetland plant. Mathematics and Computers in Simulation, 152, 69-80. 